\title{
Phytomedicinal aspects of sesquiterpenoid peroxides: Origin, structures and biological activity
}

\author{
Siddiq A ${ }^{1}$, Yaremenko I ${ }^{2}$, Terent'v AO ${ }^{2}$, Gloriozova TA ${ }^{3}$, Dzhemileva LU ${ }^{4}$, D'yakonov $\mathrm{AV}^{4}, \mathrm{Vil}^{\mathrm{V}} \mathrm{V}^{2}$ and Dembitsky $\mathrm{VM}^{2,4 *}$ \\ ${ }^{1}$ Department of Pharmacology, University of Karachi, Karachi 75270, Pakistan \\ ${ }^{2}$ N.D. Zelinsky Institute of Organic Chemistry, Russian Academy of Sciences, Leninsky Prospect 47, Moscow 119991, Russia \\ ${ }^{3}$ Institute of Biomedical Chemistry, Russian Academy of Sciences, Moscow 119121, Russia \\ ${ }^{4}$ Institute of Petrochemistry and Catalysis, Russian Academy of Sciences, Ufa 450075, Russia
}

\begin{abstract}
Sesquiterpenoid peroxides are a rare group of natural metabolites isolated predominantly from various parts of plants. The biological activity of sesquiterpene peroxides has hardly been studied. This review provides information on the biological activity of 80 natural sesquiterpene peroxides. All isolated chemical compounds are divided into two groups. The first group includes sesquiterpene hydroperoxides and the second group includes sesquiterpene lactone endoperoxides. To determine the biological activity of sesquiterpene peroxides, we used the computer PASS program, which contains more than 1,000,000 natural and synthetic chemicals that exhibit more than 10,000 different activities. As shown by the analysis of these compounds, sesquiterpene peroxides are inhibitors of cell adhesion and also demonstrate antiulcer activity. In addition, individual representatives of sesquiterpene hydroperoxides showed antineoplastic and antimetastatic activity. On the other hand, sesquiterpene lactone endoperoxides demonstrated anti-inflammatory and antiprotozoal properties. The new data presented showed that sesquiterpene peroxides are an interesting group of natural medicinal preparations that can be used in pharmacology, medicine and related fields.
\end{abstract}

\section{Introduction}

Natural sesquiterpenoids are known to consist of three isoprene units and can be acyclic or contain up to three rings including many different combinations [1-5]. These compounds are found in various parts of plants [6-17], insects [18,19], and marine algae and invertebrates $[1,2,20,21]$, and are also produced by fungal species $[22,23]$ and bacteria [24]. Sesquiterpenoid peroxides are a rare group of natural drugs, and these compounds are produced by microorganisms and are also found in marine algae and invertebrates, fungi and of plants [25-32].

To study the activity of natural sesquiterpenoid peroxides, we used the PASS program, which contains information about a million chemical compounds and more than 10,000 biological activities of both natural and synthetic compounds [33]. The algorithm for practical use of PASS has been described in detail in several publications [34-37]. In the literature, there are few such articles that link the structure of natural sesquiterpenoids and their biological activity [38-40]. As a rule, the PASS program used in $95 \%$ confirms the activity of compounds obtained by extensive in vivo or in vitro and also provides an opportunity to see a wider range of biological activities for an individual metabolite.

This review is devoted to an interesting group of natural sesquiterpenoid peroxides and the study of their predicted biological activity.

\section{Sesquiterpenoid hydroperoxides}

Acyclic terpenes as a rare group of natural hydroperoxides (16, structures are shown in Figure 1, and activity is shown in Table 1) and also the davanone type hydroperoxides, (7-12) including arteincultone (7) and epi-arteincultone (8) were found in the aerial parts of flowering plants Artemisia abrotanum [41]. Arteincultone (7) was also recovered from extracts of A. herba-alba [42] and Artemisia aucheri and it showed strong anticancer activity against the MCF-7, SK-N-MC and A2780 cell lines with IC50 values of 8.5, 9.6 and 10.9 $\mu \mathrm{g} / \mathrm{mL}$, respectively [43], and modest antimalarial activity against Plasmodium falciparum [44,45].

Hydroperoxide called litseaverticillol E (13) was detected in the extracts of the leaves and twigs of Litsea verticillata and structure was confirmed by synthesis $[46,47]$. Two bisabolane-type sesquiterpenes called peroxylippidulcine A (14) and B (15) were isolated from the aerial parts of Lippia dulcis [48]. The aerial parts of Carthamus lanatus afforded two oxygenated bisabolane fucoside (16) [49], and bisabolene derivative (17) was found from the aerial parts of Achillea clavennae [50]. The Carthamus glaucus known as the glaucous star thistle contained bisabolene fucopyranoside (18) [51]. Two isomeric sesquiterpene hydroperoxides called santalane (19) and isocampherenane (20) were isolated from a dichloromethane extract of Illicium tsangii [5254]. A eudesmane derivative hydroperoxygynuradiene (21) has been obtained from the root of Gynura bicolor [55]. The aerial parts of Aster spathulifolius was the source for cytotoxic sesquiterpene hydroperoxide, $7 \beta$-hydroperoxyeudesma-11-en-4-ol (22) [56,57], and compound (23) was discovered from two plant species Xylopia emarginata and Ecdysanthera rosea, respectively $[58,59]$.

${ }^{\star}$ Correspondence to: Valery M. Dembitsky, N.D. Zelinsky Institute of Organic Chemistry, Russian Academy of Sciences, Leninsky Prospect 47, Moscow, 119991, Russia, Tel: 791638540 80, Fax: 7(499)135 53 28, E-mail: devalery@ioc.ac.ru

Key words: hydroperoxides, endoperoxides, plant, sesquiterpenoids, activity

Received: September 18, 2019; Accepted: October 02, 2019; Published: October 10,2019 
<smiles>C=C[C@](C)(O)CCC(OO)C(C)C(=C)CC(=O)C=C(C)C</smiles><smiles>C=CC(C)(O)CC=CC(C)(O)CC(=O)C=C(C)C</smiles>

4<smiles>C=CC1(C)CCC([C@@H](C)C(=O)/C=C/C(C)(C)O)O1</smiles><smiles>C=C1C(=O)OCC1CC(OO)C(C)CC(=O)C=C(C)C</smiles>

10<smiles>CC1=C[C@H](O)[C@H](/C=C(\C)C/C=C/C(C)(C)O)C1=O</smiles><smiles>C=CC(C)(CC/C=C(\C)C(=O)/C=C/C(C)(C)O)OC(=O)O</smiles>

$\mathrm{OOH}$<smiles>C=CC(C)(O)CC(O)/C=C(\C)CCC(OO)C(=C)C</smiles><smiles>C=CC1(C)CCC([C@@H](C)C(=O)/C=C/C(C)(C)O)O1</smiles><smiles>C=C1OC(CC(C)(O)/C=C/C2COC(=O)C2=C)CC1C</smiles><smiles></smiles><smiles>C=CC(C)(O)CC/C=C(\C)C(=O)/C=C/C(C)(C)O</smiles><smiles>C=CC(C)(O)CC(O)C=C(C)CC=CC(C)(C)O</smiles><smiles>C=CC1(C)CCC2(OOC(C)(C=CC(C)(C)O)C2C)O1</smiles><smiles>C=C1OC(CC(=C)C(O)CC2COC(=O)C2=C)CC1C</smiles><smiles>C=C(C)[C@H](O)CC[C@](C)(O)[C@H]1CCC(C)CC1=O</smiles><smiles>CCO[C@](C)(/C=C\C(C)(C)O)C1CC=C(C)CC1</smiles><smiles>C=C(C)[C@H](O)C[C@](C)(OC(OC(C)C(C)O)C(C)O)[C@H]1C=C[C@@](C)(O)CC1</smiles><smiles>CC(C)(O)/C=C/CC1(C)C2C3CC4C3C4C21</smiles>

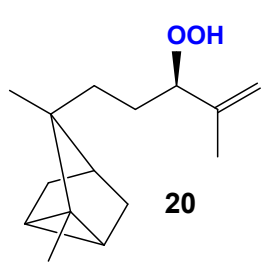<smiles>CCC(C)(O)C1=CC[C@]2(C)CCC[C@H](C)C2=C1</smiles><smiles>C=C(C)C1(O)CC[C@]2(C)CCC[C@](C)(O)[C@@]2(C)C1</smiles><smiles>C=C1CCC[C@]2(C)CC[C@H](C(C)C(=O)O)C[C@]12O</smiles>

Figure 1. Bioactive sesquiterpene hydroperoxides derived from plants

The eudesmene-type sesquiterpene peroxide called kandenol C (24, structure are shown in Figure 2, and activity is shown in Table 2) produced by Streptomyces sp. derived from the mangrove plant Kandelia candel [60]. The aerial parts of the flowering plants in the family Asteraceae, Inula japonica contained eudesmane sesquiterpenoid (25) [61]. Hydroperoxide of dihydroartemisinic acid (26) was isolated from the plant Artemisia annua (Wallaart et al., 1999), and compound (27) was isolated from leaves of Cacalia tangutica [62], and the Mexican tree Robinsonecio gerberifolius afforted hydroperoxide (28) [63]. Hydroperoxyeudesmane cinnamate (29) and compound (39) were found in extracts of Brintonia discoidea [64], and Verbesina subcordata [65].

Series of eudesmone hydroperoxides (30-35) have been found and isolated from Epaltes divericata [66], E. mexicana [67], Pluchea arguta [68], P. symphytifolia [69], and P. quitoc [70]. Hydroperoxide
(36) was isolated as an unstable oil from Artemisia herba-alba subsp. herba-alba and Ursinia tenuifolia [71], and Artemisia barrelieri [72,73]. Peroxide (37) was isolated from the aerial parts of Chrysanthemum lavandulifolium [74], and germacranolide (38) was detected in the leaves of Artemisia ludoviciana [75]. A germacranolide hydroperoxide (40) was identified from the leaves of Mulgedium tataricum [76], and the eudesmanolide (41) has been isolated from Atractylodes macrocephala [77].

Sesquiterpene lactone called tanaparthin $\alpha$-peroxide (42) was discovered in the Tanacetum parthenium [78,79], and more recently found in Achillea nobilis [80]. The 8 8,12 -guaianolide hydroperoxide (43) was obtained from Geigeria plumose [81], compound (44) was recovered from Xanthium pungens [82], and a-peroxyachifolide (45) [83]. A hydroperoxysesquiterpene lactone called crispolide (46) was isolated from the aerial parts of Tanacetum vulgare var. crispum 
Table 1. Predicted biological activity of sesquiterpenoid hydroperoxides

\begin{tabular}{|c|c|}
\hline No. & Predicted biological activity, $(\mathbf{P a})^{*}$ \\
\hline 1 & $\begin{array}{l}\text { Antiulcerative }(0,861) \text {; Cell adhesion molecule inhibitor }(0,811) \text {; Hypolipemic }(0,744) \text {; Antineoplastic }(0,711) \\
\text { Antifungal }(0,680) \text {; Apoptosis agonist }(0,645) \text {; Antiprotozoal }(0,613) \text {; Antiinflammatory }(0,595) \text {; Immunosuppressant }(0,575)\end{array}$ \\
\hline 2 & $\begin{array}{l}\text { Antineoplastic }(0,877) \text {; Apoptosis agonist }(0,786) \text {; Antiprotozoal (Plasmodium) }(0,653) \text {; Cell adhesion molecule inhibitor }(0,646) \\
\text { Antipsoriatic }(0,635) \text {; Antieczematic }(0,601) \text {; Antiulcerative }(0,600) \text {; Antiinflammatory }(0,527)\end{array}$ \\
\hline 3 & $\begin{array}{l}\text { Cell adhesion molecule inhibitor }(0,901) \text {; Antineoplastic }(0,896) \text {; Apoptosis agonist }(0,778) \text {; Antiprotozoal (Plasmodium) }(0,710) \\
\text { Antipsoriatic }(0,662) \text {; Antiulcerative }(0,651) \text {; Antieczematic }(0,627) \text {; Antiinflammatory }(0,615) \text {; Hepatic disorders treatment }(0,565)\end{array}$ \\
\hline 4 & $\begin{array}{l}\text { Cell adhesion molecule inhibitor }(0,873) \text {; Antineoplastic }(0,826) \text {; Antiulcerative }(0,789) \text {; Hepatic disorders treatment }(0,741) \\
\text { Apoptosis agonist }(0,696) \text {; Antieczematic }(0,684) \text {; Antiprotozoal }(0,676) \text {; Antiinflammatory }(0,636) \text {; Immunosuppressant }(0,621)\end{array}$ \\
\hline 5 & $\begin{array}{l}\text { Antiulcerative }(0,860) \text {; Cell adhesion molecule inhibitor }(0,824) \text {; Hypolipemic }(0,728) \text {; Antineoplastic }(0,700) \\
\text { Antiinflammatory }(0,664) \text {; Antifungal }(0,619) \text {; Gastric antisecretory }(0,542) \text {; Antiprotozoal (Plasmodium) }(0,532)\end{array}$ \\
\hline 6 & $\begin{array}{l}\text { Cell adhesion molecule inhibitor }(0,874) \text {; Antineoplastic }(0,863) \text {; Antiinflammatory }(0,728) \text {; Antiulcerative }(0,685) \\
\text { Antieczematic }(0,646) \text {; Antipsoriatic }(0,624) \text {; Hypolipemic }(0,588) \text {; Antifungal }(0,562) \text {; Apoptosis agonist }(0,561)\end{array}$ \\
\hline 7 & Antineoplastic $(0,897)$; Antimetastatic $(0,651)$; Antiprotozoal $(0,641)$; Antipsoriatic $(0,598)$; Hypolipemic $(0,582)$ \\
\hline 8 & Antineoplastic $(0,897)$; Antimetastatic $(0,651)$; Antiprotozoal $(0,641)$; Antipsoriatic $(0,598)$; Hypolipemic $(0,582)$ \\
\hline 9 & Antiprotozoal (Plasmodium) (0,914); Antineoplastic $(0,840)$; Hypolipemic $(0,693)$; Apoptosis agonist $(0,576)$ \\
\hline 10 & $\begin{array}{l}\text { Apoptosis agonist }(0,797) \text {; Antieczematic }(0,799) \text {; Antineoplastic }(0,773) \text {; Antifungal }(0,624) \text {; Antiulcerative }(0,615) \\
\text { Genital warts treatment }(0,630) \text {; Immunosuppressant }(0,602) \text {; Hypolipemic }(0,573)\end{array}$ \\
\hline 11 & $\begin{array}{l}\text { Antieczematic }(0,890) \text {; Antineoplastic }(0,880) \text {; Apoptosis agonist }(0,792) \text {; Genital warts treatment }(0,682) \\
\text { Angiogenesis stimulant }(0,672) \text {; Antipsoriatic }(0,645) \text {; Immunosuppressant }(0,604) \text {; Antifungal }(0,539) \text {; Hypolipemic }(0,512)\end{array}$ \\
\hline 12 & Antieczematic $(0,883)$; Antineoplastic $(0,810)$; Apoptosis agonist $(0,704)$; Genital warts treatment $(0,675)$ \\
\hline 13 & $\begin{array}{l}\text { Antineoplastic }(0,882) \text {; Antieczematic }(0,682) \text {; Immunosuppressant }(0,675) \text {; Carminative }(0,620) \text {; Hypolipemic }(0,610) \\
\text { Apoptosis agonist }(0,575) \text {; Antiulcerative }(0,541) \text {; Antiinflammatory }(0,538)\end{array}$ \\
\hline 14 & Antieczematic $(0,827)$; Antineoplastic $(0,804)$; Immunosuppressant $(0,619)$; Antipsoriatic $(0,614)$; Apoptosis agonist $(0,565)$ \\
\hline 15 & Antiulcerative $(0,850)$; Antieczematic $(0,751)$; Antineoplastic $(0,710)$; Immunosuppressant $(0,689)$; Apoptosis agonist $(0,638)$ \\
\hline 16 & $\begin{array}{l}\text { Antineoplastic }(0,886) \text {; Antiulcerative }(0,859) \text {; Apoptosis agonist }(0,796) \text {; Antimetastatic }(0,738) \text {; Antieczematic }(0,738) \\
\text { Vasoprotector }(0,713) \text {; Respiratory analeptic }(0,682) \text {; Antiinflammatory }(0,682) \text {; Antihelmintic }(0,666) \text {; Hepatic disorders treatment }(0,646) \text {; Antithrombotic }(0,634) \text {; Antioxidant } \\
(0,618) \text {; Antipsoriatic }(0,578) \text {; Dementia treatment }(0,552) \text {; Antifungal }(0,545)\end{array}$ \\
\hline 17 & Antiulcerative $(0,798)$; Antineoplastic $(0,731)$; Carminative $(0,657)$; Immunosuppressant $(0,654)$; Hypolipemic $(0,594)$ \\
\hline 18 & $\begin{array}{l}\text { Antiinflammatory }(0,895) \text {; Antineoplastic }(0,877) \text {; Antiulcerative }(0,761) \text {; Antimetastatic }(0,726) \text {; Antifungal }(0,706) \\
\text { Apoptosis agonist }(0,660) \text {; Antibacterial }(0,614) \text {; Antioxidant }(0,610) \text {; Antithrombotic }(0,562) \\
\text { Proliferative diseases treatment }(0,552) \text {; Antiprotozoal (Plasmodium) }(0,540)\end{array}$ \\
\hline 19 & Renin release stimulant $(0,874)$; Antineoplastic $(0,766)$; Allergic conjunctivitis treatment $(0,638)$; Nitric oxide scavenger $(0,623)$ \\
\hline 20 & Hypolipemic $(0,614)$; Antieczematic $(0,566)$; Allergic conjunctivitis treatment $(0,553)$; Antiulcerative $(0,528)$ \\
\hline 21 & $\begin{array}{l}\text { Antineoplastic }(0,710) \text {; Immunosuppressant }(0,650) \text {; Hypolipemic }(0,621) \text {; Dermatologic }(0,606) \text {; Antieczematic }(0,582) \\
\text { Antiinflammatory }(0,557) \text {; Apoptosis agonist }(0,509) \text {; Prostate disorders treatment }(0,500)\end{array}$ \\
\hline 22 & $\begin{array}{l}\text { Antineoplastic }(0,865) \text {; Immunosuppressant }(0,734) \text {; Antieczematic }(0,709) \text {; Antidiabetic symptomatic }(0,707) \\
\text { Ovulation inhibitor }(0,594) \text {; Antiprotozoal (Plasmodium) }(0,591) \text {; Prostate disorders treatment }(0,570) \text {; Antiinflammatory }(0,534)\end{array}$ \\
\hline 23 & $\begin{array}{l}\text { Antieczematic }(0,888) \text {; Antineoplastic }(0,784) \text {; Immunosuppressant }(0,697) \text {; Apoptosis agonist }(0,641) \text {; Antipsoriatic }(0,577) \\
\text { Ovulation inhibitor }(0,544) \text {; Chemoprotective }(0,516) \text {; Erythropoiesis stimulant }(0,516)\end{array}$ \\
\hline
\end{tabular}

*Only activities with $\mathrm{Pa}>0.5$ are shown

and from two T. vulgare chemotypes [84]. The 6a,12-germacranolide (47) was found in the leaves of Artemisia herba-alba [42], and peroxycostunolide (48) was detected in Tanacetum argentum [85]. Highly oxygenated sesquiterpene lactone (49) was isolated from Centaurea hermannii [86], and guaianolide (50) was found in Anthemis carpatica [87].

\section{Sesquiterpene endoperoxides}

Endoperoxy cuparene-type sesquiterpenoids (51-53, structures are shown in Figure 3, and activity is shown in Table 3) were identified from the Japanese liverwort Jungermannia infusca $[88,89]$. The chamigranes called merulin B (54) and C (55) have been found in an extract of the culture broth of a Thai mangrove-derived fungus [90,91]. Sesquiterpenoid called okundoperoxide (56) with antiplasmodial activity was detected in extracts of the plants from the family Cyperaceae,
Scleria striatinux [92]. Two muurolane sesquiterpene endoperoxides, 1,4-peroxymuurol-5-ene (57) and 1,4-peroxy-5-hydroxy-muurol-6-ene (58) have been obtained from Illicium tsangii (family Schisandraceae) [52-54]. The hydroperoxide called schisansphene A (59) was isolated from the plant Schisandra sphenanthera known as magnolia berry [93].

Highly oxygenated sesquiterpene (+)-muurolan-4,7-peroxide (60) was found in the essential oil of the liverwort Plagiochila asplenioides [94], and two sesquiterpene endoperoxides (61 and 62) were isolated from the aerial parts of the invasive plant Eupatorium adenophorum $[95,96]$. Unusual endoperoxide (63) was detected in the Ligularia veitchiana [97], compound (64) was isolated from the leaves of Eupatorium adenophorum [98], and metabolite (65) was found in extracts of the Xylopia emarginata [58]. The aerial parts of Montanoa hibiscifolia afforded rare endoperoxide (66) [99]. 


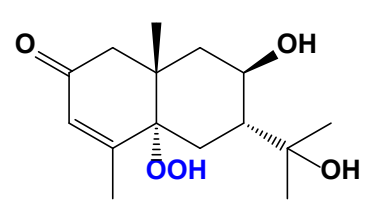

24<smiles>CC(C)O[C@H]1C[C@@H](O)C2=CC(=O)C(C(C)(C)O)=C[C@@]2(C)[C@H]1C</smiles>

28<smiles>C=C(C)[C@]1(O)C[C@H]2C(=O)C[C@](C)(CC[C@@H]2O)[C@@]1(C)O</smiles><smiles>CC1=C2[C@H](O)C[C@@H](O)[C@@H](O)[C@]2(C)CC[C@@H]1C(C)C</smiles><smiles>C=C(C[C@@H]1OC(=O)C(=C)[C@H]1C(OC)OC)[C@@H](O)CC/C(C)=C/C</smiles>

39<smiles>C=C1C(=O)OC2C[C@@]3(C)CC[C@H](O)C(=C)[C@@]3(O)CC12</smiles>

43<smiles>[Z17]O[C@H]1OC(=O)C(=C)[C@@H]1[C@H]1CC(=O)[C@@H](O)CC[C@]2(C)O[C@H]12</smiles><smiles>C=C(C(=O)OC)[C@H]1C[C@@]2(O)C(=C)CC[C@H](O)[C@]2(C)C[C@H]1OC(C)=O</smiles>

25<smiles>C[C@H]1CC[C@H](C)[C@H]2CC[C@](C)(O)CC12</smiles>

26<smiles>[Z7]C[C@]12C=C(C(C)(C)O)C(=O)C=C1CC[C@H](O)[C@@H]2C</smiles>

27<smiles>C=C1CCC[C@]2(C)CC[C@H](C(C)(C)O)C[C@]12O</smiles>

29<smiles>C=C(C)[C@@]1(O)C[C@@H]2[C@](C)(O)[C@H](OC(C)=O)CC[C@]2(C)CC(=O)[C@]1(C)O</smiles>

30<smiles>CC(=O)O[C@H]1CC[C@]2(C)CC(=O)C(C(C)(C)O)=C[C@H]2[C@]1(C)OC(C)(C)O</smiles>

31<smiles>CC1OC1(C)C(=O)O[C@@H]1CC[C@@]2(C)CC(=O)C(C(C)(C)O)=C[C@@H]2[C@]1(C)O</smiles><smiles>OO</smiles><smiles>C=C1CCC=C2C[C@@H]3OC(=O)C(=C)[C@H]3CC(O)C2C1O</smiles>

36<smiles>C=C1CCC2[C@@H](/C=C(\C)[C@@H](O)C[C@H]1O)OC(=O)[C@]2(C)O</smiles>

40<smiles>C=C1C(=O)OC2C[C@@]3(C)CC=C[C@](C)(O)[C@@]3(C)CC12</smiles>

44

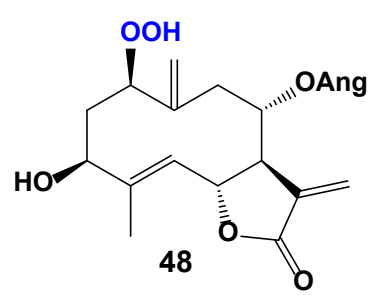<smiles>C=C1CCC[C@]2(COC(C)=O)CC3OC(=O)[C@H](C)C3CC12O</smiles>

45

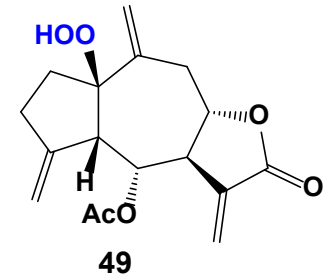

49

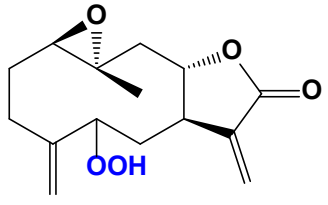

38<smiles>[Z14]C1CC2=C(C)C(=O)O[C@@]2(O)C[C@]1(C)CCCC(=C)C1CC2=C(COC(C)=O)[C@@H](O)CC[C@@]2(C)CC1OC(=O)C(=C)C</smiles>

Figure 2. Structures of sesquiterpene hydroperoxides derived from plants 
Table 2. Predicted biological activity of sesquiterpenoid hydroperoxides

\begin{tabular}{|c|c|}
\hline No. & Predicted biological activity, (Pa)* \\
\hline 24 & $\begin{array}{l}\text { Antineoplastic }(0,829) \text {; Immunosuppressant }(0,725) \text {; Antieczematic }(0,690) \text {; Allergic conjunctivitis treatment }(0,548) \\
\text { Genital warts treatment }(0,538) \text {; Dermatologic }(0,535) \text {; Antiinflammatory }(0,506)\end{array}$ \\
\hline 25 & $\begin{array}{l}\text { Antieczematic }(0,817) \text {; Antineoplastic }(0,805) \text {; Immunosuppressant }(0,742) \text {; Antiinflammatory }(0,699) \text {; Apoptosis agonist }(0,625) \\
\text { Hepatoprotectant }(0,596) \text {; Antifungal }(0,538) \text {; Antisecretoric }(0,522) \text {; Antipsoriatic }(0,519) \text {; Ovulation inhibitor }(0,513)\end{array}$ \\
\hline 26 & $\begin{array}{l}\text { Antiprotozoal (Plasmodium) }(0,785) \text {; Antineoplastic }(0,770) \text {; Antieczematic }(0,726) \text {; Immunosuppressant }(0,705) \\
\text { Antiinflammatory }(0,703) \text {; Fibrinolytic }(0,611) \text {; Prostate disorders treatment }(0,606) \text {; Antiseborrheic }(0,559)\end{array}$ \\
\hline 27 & $\begin{array}{l}\text { Antineoplastic }(0,788) \text {; Antieczematic }(0,723) \text {; Antiseborrheic }(0,706) \text {; Vasoprotector }(0,681) \text {; Immunosuppressant }(0,673) \\
\text { Antiinflammatory }(0,621) \text {; Ovulation inhibitor }(0,606) \text {; Hypolipemic }(0,592) \text {; Alopecia treatment }(0,544) \text {; Apoptosis agonist }(0,544) \\
\text { Genital warts treatment }(0,589) \text {; Antipruritic, allergic }(0,516) \text {; Endometrios treatment }(0,511) \text {; Antinephrotoxic }(0,506)\end{array}$ \\
\hline 28 & Antineoplastic $(0,857)$; Apoptosis agonist $(0,697)$; Antiinflammatory $(0,675)$; Immunosuppressant $(0,663)$; Hypolipemic $(0,629)$ \\
\hline 29 & $\begin{array}{l}\text { Antieczematic }(0,879) \text {; Antineoplastic }(0,760) \text {; Immunosuppressant }(0,736) \text {; Antiprotozoal (Plasmodium) }(0,653) \\
\text { Antipsoriatic }(0,562) \text {; Ovulation inhibitor }(0,555) \text {; Prostate disorders treatment }(0,543) \text {; Respiratory analeptic }(0,523)\end{array}$ \\
\hline 30 & $\begin{array}{l}\text { Antineoplastic }(0,904) \text {; Apoptosis agonist }(0,738) \text {; Immunosuppressant }(0,734) \text {; Antiinflammatory }(0,721) \text {; Antifungal }(0,716) \\
\text { Antieczematic }(0,649) \text {; Hepatoprotectant }(0,603) \text {; Cytostatic }(0,562) \text {; Antibacterial }(0,538) \text {; Antiprotozoal (Plasmodium) }(0,538)\end{array}$ \\
\hline 31 & $\begin{array}{l}\text { Antineoplastic }(0,821) \text {; Antiinflammatory }(0,714) \text {; Apoptosis agonist }(0,664) \text {; Antifungal }(0,650) \text {; Hypolipemic }(0,643) \\
\text { Immunosuppressant }(0,614) \text {; Cytostatic }(0,580) \text {; Hepatoprotectant }(0,578) \text {; Dermatologic }(0,557) \text {; Antibacterial }(0,550)\end{array}$ \\
\hline 32 & $\begin{array}{l}\text { Antineoplastic }(0,885) \text {; Immunosuppressant }(0,740) \text {; Antieczematic }(0,731) \text {; Antifungal }(0,601) \text {; Antiinflammatory }(0,567) \\
\text { Antidiabetic }(0,560) \text {; Apoptosis agonist }(0,556) \text {; Antiprotozoal (Plasmodium) }(0,554) \text {; Erythropoiesis stimulant }(0,547)\end{array}$ \\
\hline 33 & Antineoplastic $(0,736)$; Hypolipemic $(0,660)$; Dermatologic $(0,616)$; Immunosuppressant $(0,526)$; Cholesterol antagonist $(0,523)$ \\
\hline 34 & Renin release stimulant $(0,756)$; Antineoplastic $(0,677)$; Dermatologic $(0,624)$; General pump inhibitor $(0,550)$ \\
\hline 35 & $\begin{array}{l}\text { Antineoplastic }(0,857) \text {; Carminative }(0,725) \text {; Antieczematic }(0,714) \text {; Antiprotozoal (Plasmodium) }(0,695) \text {; Antileukemic }(0,622) \\
\text { Immunosuppressant }(0,600) \text {; Antipruritic, allergic }(0,591) \text {; Antimitotic }(0,572) \text {; Antipsoriatic }(0,544)\end{array}$ \\
\hline 36 & $\begin{array}{l}\text { Antineoplastic }(0,900) \text {; Antieczematic }(0,891) \text {; Apoptosis agonist }(0,831) \text {; Antiprotozoal (Plasmodium) }(0,827) \\
\text { Antiinflammatory }(0,705) \text {; Antileukemic }(0,649) \text {; Immunosuppressant }(0,629) \text {; Cytostatic }(0,581) \text {; Antimetastatic }(0,580)\end{array}$ \\
\hline 37 & $\begin{array}{l}\text { Antineoplastic }(0,918) \text {; Antieczematic }(0,891) \text {; Antiprotozoal (Plasmodium) }(0,868) \text {; Apoptosis agonist }(0,841) \\
\text { Antiinflammatory }(0,715) \text {; Antileukemic }(0,660) \text {; Immunosuppressant }(0,631) \text {; Antimetastatic }(0,579) \text {; Cytostatic }(0,512)\end{array}$ \\
\hline 38 & $\begin{array}{l}\text { Antineoplastic }(0,925) \text {; Antiprotozoal (Plasmodium) }(0,855) \text {; Antieczematic }(0,835) \text {; Apoptosis agonist }(0,771) \\
\text { Antiinflammatory }(0,709) \text {; Antimetastatic }(0,697) \text {; Cytostatic }(0,692) \text {; Immunosuppressant }(0,683) \text {; Antileukemic }(0,656)\end{array}$ \\
\hline 39 & $\begin{array}{l}\text { Apoptosis agonist }(0,906) \text {; Antineoplastic }(0,905) \text {; Antieczematic }(0,904) \text {; Antiprotozoal (Plasmodium) }(0,775) \\
\text { Cytostatic }(0,766) \text {; Antileukemic }(0,720) \text {; Immunosuppressant }(0,680) \text {; Antiinflammatory }(0,596) ; \text { Antimetastatic }(0,556)\end{array}$ \\
\hline 40 & $\begin{array}{l}\text { Antineoplastic }(0,918) \text {; Antieczematic }(0,897) \text {; Apoptosis agonist }(0,810) \text {; Antiprotozoal (Plasmodium) }(0,744) \\
\text { Immunosuppressant }(0,712) \text {; Antipsoriatic }(0,682) \text {; Antileukemic }(0,668) \text {; Antiinflammatory }(0,627) \text {; Cytostatic }(0,622) \\
\text { Antifungal }(0,598) \text {; Genital warts treatment }(0,589) \text {; Antibacterial }(0,527) \text {; Bone diseases treatment }(0,511)\end{array}$ \\
\hline 41 & $\begin{array}{l}\text { Antieczematic }(0,940) \text {; Antineoplastic }(0,896) \text {; Antipsoriatic }(0,701) \text {; Immunosuppressant }(0,694) \text {; Antiprotozoal (Plasmodium) }(0,679) \text {; Apoptosis agonist }(0,642) \text {; Antiosteoporotic } \\
(0,628) \text {; Ovulation inhibitor }(0,579) \text {; Prostate disorders treatment }(0,576)\end{array}$ \\
\hline 42 & $\begin{array}{l}\text { Antineoplastic }(0,862) \text {; Antieczematic }(0,845) \text {; Apoptosis agonist }(0,790) \text {; Antiprotozoal (Plasmodium) }(0,714) \\
\text { Antiinflammatory }(0,709) \text {; Immunosuppressant }(0,673) \text {; Cytostatic }(0,631) \text {; Antifungal }(0,508) \text {; Antibacterial }(0,501)\end{array}$ \\
\hline 43 & $\begin{array}{l}\text { Antieczematic }(0,856) \text {; Antineoplastic }(0,819) \text {; Antiprotozoal (Plasmodium) }(0,737) \text {; Apoptosis agonist }(0,693) \\
\text { Immunosuppressant }(0,607) \text {; Antiinflammatory }(0,603) \text {; Cytostatic }(0,582) \text {; Antimetastatic }(0,533)\end{array}$ \\
\hline 44 & $\begin{array}{l}\text { Antineoplastic }(0,891) \text {; Antieczematic }(0,822) \text {; Apoptosis agonist }(0,779) \text {; Antiinflammatory }(0,761) \text {; Immunosuppressant }(0,698) \\
\text { Antifungal }(0,667) \text {; Antiprotozoal (Plasmodium) }(0,659) \text {; Antileukemic }(0,526) \text {; Dermatologic }(0,526)\end{array}$ \\
\hline 45 & $\begin{array}{l}\text { Antieczematic }(0,838) \text {; Antineoplastic }(0,829) \text {; Antiprotozoal (Plasmodium) }(0,749) \text {; Immunosuppressant }(0,726) \\
\text { Cytostatic }(0,724) \text {; Antiinflammatory }(0,710) \text {; Antiulcerative }(0,662) \text {; Apoptosis agonist }(0,607) \text {; Antimetastatic }(0,603) \\
\text { Antifungal }(0,585) \text {; Antileukemic }(0,527) \text {; Antihelmintic }(0,510) \text {; Antipsoriatic }(0,507)\end{array}$ \\
\hline 46 & $\begin{array}{l}\text { Antineoplastic }(0,939) \text {; Antieczematic }(0,863) \text {; Antiinflammatory }(0,804) \text {; Antimitotic }(0,776) \text {; Immunosuppressant }(0,655) \\
\text { Antiprotozoal (Plasmodium) }(0,650) \text {; Apoptosis agonist }(0,612) \text {; Genital warts treatment }(0,600) \text {; Prostate disorders treatment }(0,570)\end{array}$ \\
\hline 47 & $\begin{array}{l}\text { Antineoplastic }(0,951) \text {; Cytostatic }(0,896) \text {; Antieczematic }(0,881) \text {; Apoptosis agonist }(0,880) \text {; Antiprotozoal (Plasmodium) }(0,845) \\
\text { Antileukemic }(0,829) \text {; Immunosuppressant }(0,726) \text {; Antiinflammatory }(0,720) \text {; Antimetastatic }(0,665) \text {; Antiparasitic }(0,655) \\
\text { Antipsoriatic }(0,652) \text {; Antifungal }(0,596) \text {; Antibacterial }(0,590) \text {; Prostate cancer treatment }(0,541)\end{array}$ \\
\hline 48 & $\begin{array}{l}\text { Antineoplastic }(0,945) \text {; Antieczematic }(0,919) \text {; Apoptosis agonist }(0,915) \text {; Cytostatic }(0,904) \text {; Antileukemic }(0,796) \\
\text { Antiinflammatory }(0,778) \text {; Antiprotozoal (Plasmodium) }(0,772) \text {; Antifungal }(0,761) \text {; Antiparasitic }(0,749) \text {; Antipsoriatic }(0,726) \\
\text { Immunosuppressant }(0,699) \text {; Antibacterial }(0,689) \text {; DNA synthesis inhibitor }(0,503)\end{array}$ \\
\hline 49 & $\begin{array}{l}\text { Antineoplastic }(0,955) \text {; Apoptosis agonist }(0,885) \text {; Antieczematic }(0,843) \text {; Cytostatic }(0,723) \text {; Antiprotozoal (Plasmodium) }(0,718) \\
\text { Immunosuppressant }(0,714) \text {; Antiinflammatory }(0,710) \text {; Antimitotic }(0,684) \text {; Antileukemic }(0,582) \text {; Antifungal }(0,521)\end{array}$ \\
\hline 50 & $\begin{array}{l}\text { Antineoplastic }(0,903) \text {; Antieczematic }(0,798) \text {; Antiinflammatory }(0,711) \text {; Immunosuppressant }(0,644) \text {; Antifungal }(0,632) \\
\text { Antiprotozoal (Plasmodium) }(0,591) \text {; Apoptosis agonist }(0,585) \text {; Cytostatic }(0,527)\end{array}$ \\
\hline
\end{tabular}

*Only activities with $\mathrm{Pa}>0.5$ are shown 

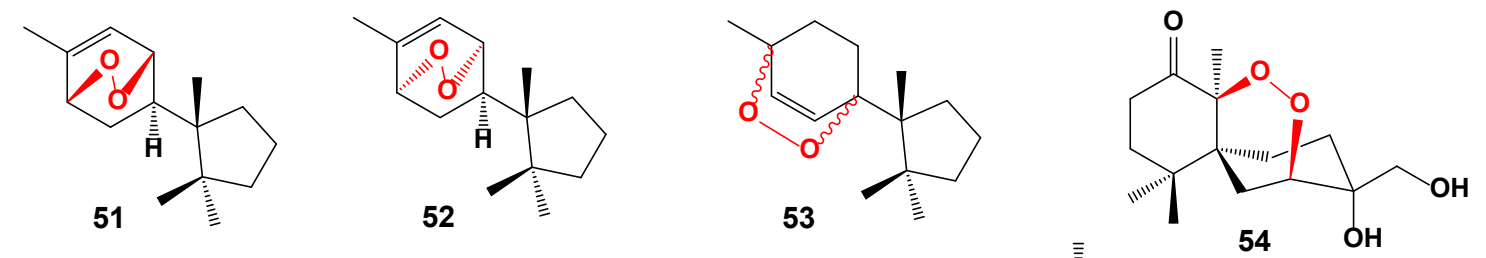<smiles>CC1(C)CCC(=O)[C@@]2(C)OO[C@H]3C[C@]12CC3(O)CO</smiles><smiles>C/C(=C\CO)[C@H]1C[C@H]2C(C)(C)C(=O)C=C[C@@]2(C)OO1</smiles><smiles>[B]C(C)[C@H]1CC[C@@H](C)C23C=CC(C)(C=C12)O3</smiles>

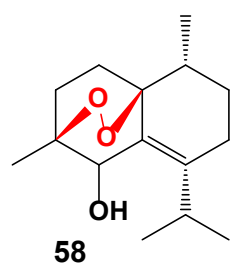<smiles>C[C@@H]1CCC[C@@]2(C)CCC(C(C)(C)O)=C[C@]12OO</smiles><smiles>CC(C)[C@H]1CC[C@@H](C)[C@]2(O)C[C@]3(C)CC[C@@H]1[C@]32O</smiles><smiles>CC1=C[C@]23O[C@](O)(C[C@@H](C)[C@@H]2CC1=O)[C@H](C(C)C)C3(O)O</smiles><smiles>[R6]C(OO[C@]1(C)CC[C@H]2[C@@H](C)CC[C@]2(C)C1)C(C)C</smiles><smiles></smiles>

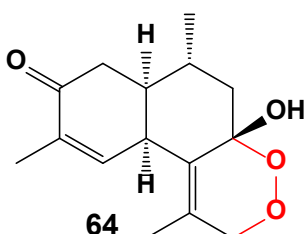<smiles>C[C@H]1CC[C@@]2(C)C3CC[C@](O)(CO3)C2C1</smiles>

<smiles>C=C1C(=O)O[C@H]2C[C@H](C)C34CCC(C)(OO3)[C@H](C[C@@H]12)[C@H]4Br</smiles><smiles>C[C@H]1C(=O)O[C@H]2C1C[C@@]1(C)[C@H](O)CC[C@]13C[C@@]2(O)OO3</smiles><smiles>CC(C)[C@@]12CC[C@@](C)(OO1)[C@H](O)C1=C2C(=O)C[C@H]1[18O]</smiles><smiles>CC(C)[C@@]12OO[C@](C)(C[C@H](O)[C@@H]1O)C1=C2C(=O)C[C@H]1O</smiles><smiles>CC(C)[C@@H]1C[C@@H]2[C@@H]3OOC[C@@H]3[C@@H]2[C@@H](O)[C@H](O)C1</smiles>

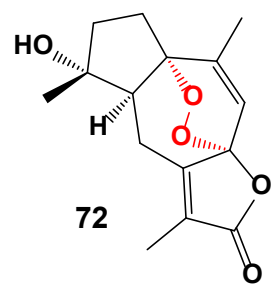

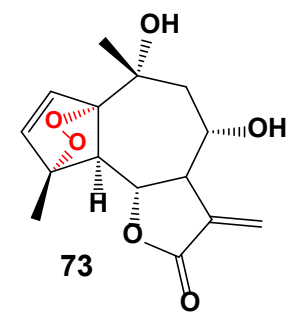

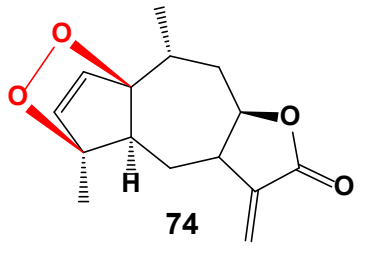<smiles>C=C1CC[C@H]2[C@@H](C)CC[C@@H]3[C@H]2[C@@H]1OOC(=O)[C@@H]3C</smiles><smiles></smiles><smiles>CC(C)=CCC[C@@H](C)C12C=CC(C)(CC1)O2</smiles><smiles>CC(C)=CCC[C@@H](C)C12C=CC(C)(CC1)OO2</smiles><smiles>C[C@H]1C(=O)O[C@@H]2C[C@@]3(O)CC4(CC[C@H](O)[C@]4(C)CCC21)OO3</smiles>

79 Tehranolide

Figure 3. Bioactive sesquiterpene endoperoxides derived from plants and fungal endophytes 
Table 3. Predicted biological activity of sesquiterpenoid endoperoxides

\begin{tabular}{|c|c|}
\hline No. & Predicted biological activity, $(\mathbf{P a})^{*}$ \\
\hline 51 & $\begin{array}{l}\text { Antiinflammatory }(0,958) \text {; Antiprotozoal (Plasmodium) }(0,936) \text {; Antineoplastic }(0,932) \text {; Antieczematic }(0,634) \\
\text { Antifungal (Candida) }(0,630) \text {; Apoptosis agonist }(0,617) \text {; Cardiovascular analeptic }(0,557) \text {; Gynecological disorders treatment }(0,509)\end{array}$ \\
\hline 52 & $\begin{array}{l}\text { Antiinflammatory }(0,958) \text {; Antiprotozoal (Plasmodium) }(0,936) \text {; Antineoplastic }(0,932) \text {; Antieczematic }(0,634) \\
\text { Antifungal (Candida) }(0,630) \text {; Apoptosis agonist }(0,617) \text {; Cardiovascular analeptic }(0,557)\end{array}$ \\
\hline 53 & $\begin{array}{l}\text { Antiprotozoal (Plasmodium) }(0,954) \text {; Apoptosis agonist }(0,910) \text {; Antineoplastic }(0,768) \text {; Antimetastatic }(0,587) \\
\text { Antiparasitic }(0,553) \text {; Antidyskinetic }(0,547) \text {; Dermatologic }(0,523) \text {; Atherosclerosis treatment }(0,520) \text {; Ovulation inhibitor }(0,517)\end{array}$ \\
\hline 54 & Antiprotozoal (Plasmodium) $(0,928)$; Antineoplastic $(0,681)$; Antiinflammatory $(0,544)$; Genital warts treatment $(0,516)$ \\
\hline 55 & Antiprotozoal (Plasmodium) $(0,928)$; Antineoplastic $(0,681)$; Antiinflammatory $(0,544)$; Genital warts treatment $(0,516)$ \\
\hline 56 & Antiprotozoal (Plasmodium) (0,951); Antineoplastic $(0,730)$; Antieczematic $(0,659)$; Antifungal $(0,500)$ \\
\hline 57 & $\begin{array}{l}\text { Antiprotozoal (Plasmodium) }(0,908) \text {; Carminative }(0,788) \text {; Antineoplastic }(0,714) \text {; Antieczematic }(0,587) \text {; Antimetastatic }(0,584) \\
\text { Antiparasitic }(0,565) \text {; Antiinflammatory }(0,540) \text {; Antiviral (Arbovirus) }(0,524) \text {; Antidyskinetic }(0,517) \text {; Antihelmintic }(0,514)\end{array}$ \\
\hline 58 & $\begin{array}{l}\text { Antiinflammatory }(0,934) \text {; Antiprotozoal (Plasmodium) }(0,879) \text {; Antineoplastic }(0,866) \text {; Antieczematic }(0,775) \\
\text { Antiparasitic }(0,649) \text {; Antimetastatic }(0,623) \text {; Antihelmintic }(0,609) \text {; Fibrinolytic }(0,582) \text {; Genital warts treatment }(0,571)\end{array}$ \\
\hline 59 & $\begin{array}{l}\text { Antiinflammatory }(0,945) \text {; Antiprotozoal (Plasmodium) }(0,916) \text {; Antineoplastic }(0,854) \text {; Antieczematic }(0,700) \\
\text { Cardiovascular analeptic }(0,651) \text {; Antifungal }(0,544) \text {; Dementia treatment }(0,540) \text {; Antiseborrheic }(0,514) \text {; Antinephrotoxic }(0,513)\end{array}$ \\
\hline 60 & $\begin{array}{l}\text { Antiprotozoal (Plasmodium) }(0,965) \text {; Antineoplastic }(0,792) \text {; Antieczematic }(0,665) \text {; Carminative }(0,652) \text {; Antimetastatic }(0,584) \\
\text { Antiparasitic }(0,576) \text {; Dermatologic }(0,537) \text {; Antileukemic }(0,519) \text {; Ovulation inhibitor }(0,518) \text {; Antinephrotoxic }(0,503)\end{array}$ \\
\hline 61 & $\begin{array}{l}\text { Antiprotozoal (Plasmodium) }(0,954) \text {; Carminative }(0,832) \text {; Antieczematic }(0,794) \text {; Antischistosomal }(0,566) \text {; Apoptosis agonist }(0,565) \text {; Antifungal }(\text { Cryptococcus })(0,549) \text {; } \\
\text { Allergic conjunctivitis treatment }(0,547) \text {; Antifungal }(0,533)\end{array}$ \\
\hline 62 & $\begin{array}{l}\text { Antiprotozoal (Plasmodium) }(0,956) \text {; Antieczematic }(0,700) \text {; Antineoplastic }(0,670) \text {; Antifungal }(0,593) \text {; Antimetastatic }(0,587) \\
\text { Antiparasitic }(0,574) \text {; Analgesic }(0,541) \text {; Antinephrotoxic }(0,520) \text {; Carminative }(0,514) \text {; Antibacterial }(0,510)\end{array}$ \\
\hline 63 & Antiprotozoal (Plasmodium) $(0,964)$; Apoptosis agonist $(0,862)$; Antineoplastic $(0,694)$; Antiseborrheic $(0,576)$ \\
\hline 64 & Antiprotozoal (Plasmodium) $(0,945)$; Antieczematic $(0,715)$; Carminative $(0,599)$; Antineoplastic $($ sarcoma $)(0,529)$ \\
\hline 65 & $\begin{array}{l}\text { Antiprotozoal (Plasmodium) }(0,881) \text {; Antieczematic }(0,734) \text {; Immunosuppressant }(0,705) \text {; Cardiovascular analeptic }(0,687) \\
\text { Antipruritic }(0,644) \text {; Antidyskinetic }(0,628) \text {; Antifungal }(0,603) \text {; Antineoplastic }(0,599) \text {; Antinephrotoxic }(0,598) \\
\text { Spasmolytic, urinary }(0,576) \text {; Dementia treatment }(0,570) \text {; Antiseborrheic }(0,545) \text {; Vasoprotector }(0,536)\end{array}$ \\
\hline 66 & $\begin{array}{l}\text { Antiprotozoal (Plasmodium) }(0,884) \text {; Antineoplastic }(0,862) \text {; Antieczematic }(0,861) \text {; Apoptosis agonist }(0,795) \\
\text { Antiinflammatory }(0,679) \text {; Antiparasitic }(0,672) \text {; Cytostatic }(0,667) \text {; Antifungal }(0,565) \text {; Antileukemic }(0,539)\end{array}$ \\
\hline 67 & $\begin{array}{l}\text { Antiprotozoal (Plasmodium) }(0,967) \text {; Antineoplastic }(0,911) \text {; Apoptosis agonist }(0,883) \text {; Antieczematic }(0,836) \\
\text { Antifungal }(0,812) \text {; Antiparasitic }(0,811) \text {; Antiprotozoal (Leishmania) }(0,731) \text {; Antiinflammatory }(0,725) \text {; Immunosuppressant }(0,675) \\
\text { Antischistosomal }(0,668) \text {; Antibacterial }(0,667) \text {; Antihelmintic }(0,664) \text {; DNA synthesis inhibitor }(0,652)\end{array}$ \\
\hline 68 & $\begin{array}{l}\text { Antiprotozoal (Plasmodium) }(0,925) \text {; Antineoplastic }(0,914) \text {; Antieczematic }(0,851) \text {; Cardiovascular analeptic }(0,775) \\
\text { Antiparasitic }(0,741) \text {; Antinephrotoxic }(0,715) \text {; Antihelmintic }(0,702) \text {; Antiinflammatory }(0,702) \text {; Immunosuppressant }(0,667) \text {; Genital warts treatment }(0,660) \text {; Antiviral } \\
\text { (Arbovirus) }(0,641) \text {; Cytostatic }(0,616) \text {; Dementia treatment }(0,539)\end{array}$ \\
\hline 69 & $\begin{array}{l}\text { Analgesic }(0,883) \text {; Carminative }(0,860) \text {; Antineoplastic }(0,787) \text {; Antiprotozoal (Plasmodium) }(0,739) \text {; Antieczematic }(0,678) \\
\text { Antiparasitic }(0,662) \text {; Antihelmintic }(0,627) \text {; Antiseborrheic }(0,617) \text {; Genital warts treatment }(0,600) \text {; Antimetastatic }(0,591)\end{array}$ \\
\hline 70 & $\begin{array}{l}\text { Antineoplastic }(0,855) \text {; Analgesic }(0,843) \text {; Carminative }(0,841) \text {; Antiprotozoal (Plasmodium) }(0,739) \text {; Antieczematic }(0,654) \\
\text { Antiinflammatory }(0,648) \text {; Prostate cancer treatment }(0,641) \text {; Apoptosis agonist }(0,594) \text {; Genital warts treatment }(0,583)\end{array}$ \\
\hline 71 & $\begin{array}{l}\text { Antiprotozoal (Plasmodium) (0,917); Antineoplastic }(0,797) \text {; Antieczematic }(0,768) \text {; Carminative }(0,724) \text {; Antiinflammatory }(0,697) \\
\text { Prostate cancer treatment }(0,650) \text {; Antifungal }(0,639) \text {; Immunosuppressant }(0,609) \text {; Genital warts treatment }(0,583)\end{array}$ \\
\hline 72 & $\begin{array}{l}\text { Antiprotozoal (Plasmodium) }(0,889) \text {; Antineoplastic }(0,769) \text {; Antieczematic }(0,651) \text {; Angiogenesis stimulant }(0,644) \\
\text { Allergic conjunctivitis treatment }(0,574) \text {; Antibacterial }(0,542) \text {; Antifungal }(0,528) \text {; Ovulation inhibitor }(0,522)\end{array}$ \\
\hline 73 & $\begin{array}{l}\text { Antiprotozoal (Plasmodium) }(0,936) \text {; Antineoplastic }(0,912) \text {; Apoptosis agonist }(0,890) \text {; Cytostatic }(0,870) \text {; Antieczematic }(0,831) \\
\text { Antiparasitic }(0,774) \text {; Antiinflammatory }(0,770) \text {; Antifungal }(0,687) \text {; Chemopreventive }(0,671) \text {; Antibacterial }(0,656)\end{array}$ \\
\hline 74 & $\begin{array}{l}\text { Antiprotozoal (Plasmodium) }(0,939) \text {; Antineoplastic }(0,898) \text {; Antieczematic }(0,825) \text {; Apoptosis agonist }(0,741) \text {; Antiparasitic }(0,716) \\
\text { Antiinflammatory }(0,690) \text {; Antifungal }(0,646) \text {; Angiogenesis inhibitor }(0,609) \text {; Antibacterial }(0,576) \text {; Antihelmintic }(0,558)\end{array}$ \\
\hline 75 & $\begin{array}{l}\text { Antiprotozoal (Plasmodium) }(0,933) \text {; Antieczematic }(0,919) \text {; Antineoplastic }(0,885) \text {; Antiinflammatory }(0,713) \\
\text { Antifungal }(0,690) \text {; Cardiovascular analeptic }(0,657) \text {; Immunosuppressant }(0,637) \text {; Ovulation inhibitor }(0,636) \\
\text { Antipsoriatic }(0,630) \text {; Prostate disorders treatment }(0,588) \text {; Antinephrotoxic }(0,579) \text {; Antimetastatic }(0,568)\end{array}$ \\
\hline 76 & $\begin{array}{l}\text { Antiinflammatory }(0,949) \text {; Antineoplastic }(0,946) \text {; Antieczematic }(0,896) \text {; Apoptosis agonist }(0,782) \text {; Antiprotozoal }(\text { Plasmodium })(0,752) \text {; Antipsoriatic }(0,678) \text {; Alopecia } \\
\text { treatment }(0,665) \text {; Respiratory analeptic }(0,643) \text {; Neuroprotector }(0,638) \\
\text { Hepatoprotectant }(0,632) \text {; Immunosuppressant }(0,608) \text {; Ovulation inhibitor }(0,602) \text {; Dementia treatment }(0,533)\end{array}$ \\
\hline 77 & $\begin{array}{l}\text { Antiprotozoal (Plasmodium) }(0,842) \text {; Antihelmintic }(0,784) \text {; Antiparasitic }(0,783) \text {; Antieczematic }(0,709) \text {; Antiviral (Arbovirus) }(0,645) \text {; Antimetastatic }(0,628) \text {; Hypolipemic } \\
(0,603) \text {; Antifungal }(0,533) \text {; Antineoplastic }(0,528)\end{array}$ \\
\hline 78 & $\begin{array}{l}\text { Antiprotozoal (Plasmodium) }(0,842) \text {; Antihelmintic }(0,784) \text {; Antiparasitic }(0,783) \text {; Antieczematic }(0,709) \text {; Antiviral (Arbovirus) }(0,645) \text {; Antimetastatic }(0,628) \text {; Hypolipemic } \\
(0,603) \text {; Antifungal }(0,533) \text {; Antineoplastic }(0,528)\end{array}$ \\
\hline 79 & $\begin{array}{l}\text { Antiprotozoal (Plasmodium) }(0,922) \text {; Antineoplastic }(0,917) \text {; Cardiovascular analeptic }(0,895) \text {; Antieczematic }(0,869) \\
\text { Antiparasitic }(0,746) \text {; Antiinflammatory }(0,726) \text {; Antihelmintic }(0,696) \text {; Antinephrotoxic }(0,695) \text {; Dementia treatment }(0,541)\end{array}$ \\
\hline
\end{tabular}

*Only activities with $\mathrm{Pa}>0.5$ are shown 
The xanthane-type sesquiterpenoid, $4 \beta, 5 \beta$-epoxyxanthatin$1 \alpha, 4 \alpha$-endoperoxide (67) was found in the aerial parts of Xanthium strumarium [100], and sesquiterpene lactone (68) was detected in the extract of the aerial parts of Artemisia diffusa [101]. An antimalarial guaiane endoperoxide, nardoperoxide (69) and related endoperoxide (70) were isolated from the roots of Nardostachys chinensis [102,103], and the sesquiterpene peroxide (71) has been found from the aerial parts of Croton arboreous [104]. Endoperoxide, $1 \alpha, 8 \alpha$-epidioxy- $4 \alpha-$ hydroxy-5aH-guai-7(11),9-dien-12,8-olide (72) was isolated from Curcuma wenyujin with anti-influenza viral activity [105].

The aerial parts of Achillea setacea afforded endoperoxide (73) [106]. The flowering plant from the sunflower family, Pulicaria undulata contained sesquiterpene peroxy lactone (74) [107]. Artemisia annua afforded a rare seven-membered endoperoxide lactone arteannuin $\mathrm{H}$ (75) $[108,109]$, and allohimachalane peroxide (76) has been obtained from Illicium tsangii [52-54].

Two diastereoisomers of a sesquiterpene endoperoxide 3,6-epidioxy-1,10-bisaboladiene (77 and 78) were obtained from the both essential oil and dichloromethane extract of the aerial parts of Senecio ventanensis [110].

An unusual sesquiterpene lactone with endoperoxide group, called tehranolide (79) with strong antimalarial activity has been discovered in many Iranian Artemisia species: A. aucheri, A. austriaca, A. biennis, A. campestris, A. deserti, A. diffusa, A. gypsacea, A. haussknechtii, A. kermanensis, A. kopetdaghensis, A. kulbadica, A. oliveriana, A. persica, A. santolina, A. sieberi, A. tschernieviana, A. ciniformis, A. incana, A. turanica and A. tournefortiana [111].

\section{Conclusion}

The presented review is devoted to a rare group of sesquiterpenoid peroxides, which are found in various parts of plants or fungal endophytes isolated from plants are produced. This group contains hydroperoxides and endoperoxides which demonstrate a wide range of biological activities. So, hydroperoxides show predominantly antitumor and antiulcer activity, and antiprotozoal and anti-inflammatory activities are characteristic for endoperoxides.

\section{Conflicts of interest}

We wish to confirm that there are no known conflicts of interest associated with this publication and there has been no significant financial support for this work that could have influenced its outcome.

\section{References}

1. Fraga BM (2013) Natural sesquiterpenoids. Nat Prod Rep 30: 1226-1264. [Crossref]

2. Fraga BM (2012) Natural sesquiterpenoids. Nat Prod Rep 29: 1334-1366. [Crossref]

3. Sharma A, Bajpai VK, Shukla S (2013) Sesquiterpenes and Cytotoxicity. In: Ramawat $\mathrm{K}$, Mérillon JM. (Eds) Natural Products. Springer, Berlin, Heidelberg.

4. Qu Z, Bendena WG, Tobe SS, Hui JHL (2018) Juvenile hormone and sesquiterpenoids in arthropods: Biosynthesis, signaling, and role of MicroRNA. J Steroid Biochem Mol Biol 184: 69-76. [Crossref]

5. Li D, Wang KW (2016) Natural new sesquiterpenes: Structural diversity and bioactivity. Current organic chemistry 20: 994-1042.

6. Chadwick M, Trewin H, Gawthrop F, Wagstaff C (2013) Sesquiterpenoids lactones: benefits to plants and people. International Journal of Molecular Science 14: 1278012805.

7. Quintana J, Estévez F (2018) Recent advances on cytotoxic sesquiterpene lactones. Curr Pharm Des 24: 4355-4361. [Crossref]
8. Castro ES, Alves A, Livia A, Lobo J, Felipe R, et al. (2017) Antileukemic properties of sesquiterpene lactones: A systematic review. Anti-Cancer Agents in Medicinal Chemistry 18: 323-334.

9. Muraleedharan KM, Avery MA (2009) Progress in the development of peroxide-based anti-parasitic agents. Drug Discov Today 14: 793-803. [Crossref]

10. Jefford CW (2007) New developments in synthetic peroxidic drugs as artemisinin mimics. Drug Discovery Today 12: 487-495.

11. Mouthé Happi G, Tchaleu Ngadjui B, Green IR, Fogué Kouam S (2018) Phytochemistry and pharmacology of the genus Entandrophragma over the 50 years from 1967 to 2018: a 'golden' overview. J Pharm Pharmacol 70: 1431-1460. [Crossref]

12. Shang ZP, Wang F, Zhang JY, Wang ZJ, Lu JQ, et al. (2017) The genus Liriope Phytochemistry and pharmacology. Chin J Nat Med 15: 801-815. [Crossref]

13. Wang AR, Song HC, An HM, Huang Q, Luo X, et al. (2015) Secondary metabolites of plants from the genus chloranthus: chemistry and biological activities. Chemistry and Biodiversity 12:451-473.

14. Ghader B, Azadeh A, Sina A, Yusof R, Shiva GGA (2018) Application of sesquiterpene lactone: A new promising way for cancer therapy based on anticancer activity. Biomedicine and Pharmacotherapy 106: 239-246.

15. Hussain H, Al-Harrasi A, Abbas G, Rehman NU, Mabood F, et al. (2013) The genus Pluchea: phytochemistry, traditional uses, and biological activities. Chemistry and Biodiversity 10:1944-1971.

16. Dembitsky VM (2004) Chemistry and biodiversity of the biologically active natural glycosides. Chem Biodivers 1: 673-781. [Crossref]

17. Hanson JR (2009) The biosynthesis of some sesquiterpenoids. Pure and Applied Chemistry 53: 1155-1162.

18. Wu Q, Sun J, Chen J, Zhang H, Guo YW, et al. (2018) Terpenoids from marine soft coral of the genus Lemnalia: Chemistry and biological activities. Marine Drugs 16: 320 .

19. Nouzova M, Rivera-Pérez C, Noriega FG (2018) Omics approaches to study juvenile hormone synthesis. Curr Opin Insect Sci 29: 49-55. [Crossref]

20. Rachel L, Yamthe T, Appiah-Opong R, Valere P, Fokou T, et al. (2017) Marine algae as source of novel antileishmanial. Drugs: A Review. Marine Drugs 15: 323.

21. Falkenberg M, Nakano E, Zambotti-Villela L, Zatelli GA, Philippus AC, et al. (2019) Bioactive compounds against neglected diseases isolated from macroalgae: a review. Journal of Applied Phycology 31: 797-823.

22. Chen HP, Liu JK (2017) Secondary metabolites from higher fungi. In: Kinghorn A Falk H, Gibbons S, Kobayashi J. (Eds) Progress in the Chemistry of Organic Natural Products Vol. 106, Springer, Cham.

23. Kramer R, Abraham WR (2012) Volatile sesquiterpenes from fungi: what are they good for? Phytochemistry Reviews 11: 15-37.

24. Yamada Y, Kuzuyama T, Komatsu M, Shin-Ya K, Omura S, et al. (2015) Terpene synthases are widely distributed in bacteria. Proc Natl Acad Sci U S A 112: 857-862. [Crossref]

25. Dembitsky VM (2008) Bioactive peroxides as potential therapeutic agents. European Journal of Medicinal Chemistry 43: 223-251.

26. Dembitsky V, Shkrob I, Hanus LO (2008) Ascaridole and related peroxides from the genus Chenopodium. Biomed Pap Med Fac Univ Palacky Olomouc Czech Repub. 152: 209-215. [Crossref]

27. Dembitsky VM, Gloriozova TA, Poroikov VV (2007) Natural peroxy anticancer agents. Mini Reviews in Medicinal Chemistry 7: 571-589. [Crossref]

28. Dembitsky VM (2015) Astonishing diversity of natural peroxides as potential therapeutic agents. J Mol Genet Med 9: 163.

29. Dembitsky VM (2015) Bioactive fungal endoperoxides. Med Mycology 1: 1-7.

30. Savidov N, Gloriozova TA, Poroikov VV, Dembitsky VM (2018) Highly oxygenated isoprenoid lipids derived from fungi and fungal endophytes: Origin and biological activities. Steroids 140: 114-124. [Crossref]

31. Vil VA, Gloriozova TA, Poroikov VV, Terent'ev AO, Savidov N, et al. (2018) Peroxy steroids derived from plant and fungi and their biological activities. Appl Microbiol Biotechnol 102: 7657-7667. [Crossref]

32. Dembitsky VM, Tolstikov AG, Tolstikov GA (2003) Natural halogenated non-terpenic C15-acetogenins of sea organisms. Chemistry for Sustainable Development 11: 329339. 
33. Filimonov DA, Lagunin AA, Gloriozova TA, Rudik AV, Druzhilovskiy DS, et al. (2014) Prediction of the biological activity spectra of organic compounds using the PASS online web resource. Chem Heterocycl Compd 50: 444-457.

34. Filimonov DA, Druzhilovskiy DS, Lagunin AA, Gloriozova TA, Rudik AV, et al. (2018) Computeraided prediction of biological activity spectra for chemical compounds: opportunities and limitations. Biom Chem Res Method 1: e00004.

35. Dembitsky VM, Savidov N, Gloriozova TA (2018) Sulphur containing steroids: Structures and biological activities. Vietnam J Chem 56: 582-540.

36. Vil VA, Gloriozova TA, Poroikov VV, Terent'ev AO, Savidov N, et al. (2019) Naturally occurring of a,ß-diepoxy-containing compounds: origin, structures, and biological activities. Applied Microbiology and Biotechnology 103: 3249-3264.

37. Vil V, Terent'ev AO, Al Quntar AAA, Gloriozova TA, Savidov N, et al. (2019) Oxetane-containing metabolites: origin, structures and biological activities. Applied Microbiology and Biotechnology 103: 2449-2467.

38. Wang GW, Qin JJ, Cheng XR, Shen YH, Shan L, et al. (2014) Inula sesquiterpenoids: structural diversity, cytotoxicity and anti-tumor activity. Expert Opin Investig Drugs 23: 317-345.

39. Durán-Peña MJ, Botubol Ares JM, Hanson JR, Collado IG, Hernández-Galán R (2015) Biological activity of natural sesquiterpenoids containing a gem-dimethylcyclopropane unit. Natural Products Repeports 32: 1236-1248.

40. Liao SG, Yue JM (2016) Dimeric sesquiterpenoids. Progress in the Chemistry of Organic Natural Products. Springer 101: 1-112.

41. Rücker G, Manns D, Wilbert S (1993) Peroxide als Pflanzeninhaltsstoffe, 12. Mitt.: Peroxide vom Davanon-Typ aus der Eberraute (Artemisia abrotanum) und ihre Darstellung. Archiv der Pharmazia 326: 457-460.

42. Marco JA, Sanz-Cervera JF, Ocete G (1994) New germacranolides and eudesmanolides from north African Artemisia herba-alba. Journal of Natural Products 57: 939-946.

43. Hosseinzadeh L, Shokoohinia Y, Arab M, Allahyari E, Mojarra M (2019) Cytotoxic and apoptogenic sesquiterpenoids from the petroleum ether extract of Artemisia aucheri aerial parts. Iranian Journal of Pharmaceutical Research 18: 391-399.

44. Rücker G (1996) Malariawirksame Verbindungen aus Pflanzen, insbesondere Peroxide. Pharmazi Userer Zeit 24: 189-195.

45. Rücker G, Walter RD, Manns D, Mayer R (1991) Antimalarial activity of some natura peroxides. Planta Medica 57: 295-297.

46. Zhang HJ, Tan GT, Santarsiero BD, Mesecar AD, Hung NV, et al. (2003) New sesquiterpenes from Litsea verticillata. Journal of Natural Products 66: 609-615.

47. Vassilikogiannakis G, Margaros I, Montagnon T (2004) Biomimetic total synthesis of litseaverticillols B, E, I, and J and structural reassignment of litseaverticillol E. Organic Letters 6: 2039-2042. [Crossref]

48. Ono M, Tsuro T, Abe H, Eto M, Okawa M, et al. (2006) Bisabolane-type sesquiterpenes from the aerial parts of Lippia dulcis. Journal of Natural Products 69: 1417-1420.

49. Mikhova M, Duddeck H, Taskova R, Mitova M, Alipieva K (2004) Oxygenated bisabolane fucosides from Carthamus lanatus L. Zeitschrift für Naturforschung C 59: 244-249. [Crossref]

50. Trifunovic S, Vajs V, Juranic Z, Zizak Z, Tesevic V, et al. (2006) Cytotoxic constituents of Achillea clavennae from Montenegro. Phytochemistry 67: 887-893.

51. Taglialatela-Scafati O, Pollastro F, Cicione L, Chianese G, Bellido ML, et al. (2012) STAT-3 Inhibitory Bisabolanes from Carthamus glaucus. J Nat Prod 75: 453-458.

52. Ngo KS, Brown GD (1999) Allohimachalane, seco-allohimachalane and himachalane sesquiterpenes from Illicium tsangii. Tetrahedron 55: 759-766.

53. Ngo KS, Brown GD (1999) Santalane and isocampherenane sesquiterpenoids from Illicium tsangii. Phytochemistry 50: 1213-1219.

54. Ngo KS, Wong WT, Brown GD (1999) Muurolane sesquiterpenes from Illicium tsangii. Journal of Natural Products 62: 549-556. [Crossref]

55. Shimizu Y, Imayoshi Y, Kato M, Maeda K, Iwabuchi H, et al. (2011) New eudesmanetype sesquiterpenoids and other volatile constituents from the roots of Gynura bicolor DC. Flavour and Fragrance Journal 26: 55-61.

56. Lee SO, Choi SZ, Choi SU, Kim GH, Kim Y C, et al. (2006) Cytotoxic terpene hydroperoxides from the aerial parts of Aster spathulifolius. Arch Pharm Res 29: 845848. [Crossref]

57. Choi SZ, Lee SO, Choiand SU, Lee KR (2003) A new sesquiterpene hydroperoxide from the aerial parts of Aster oharai. Archives of Pharmacal Research 26: 521-527.
58. Moreira IC, Roque NF, Contini K, Lago JHG (2007) Sesquiterpenes and hydrocarbons from Xylopia emarginata (Annonaceae) fruits. Revista Brasileira de Farmacognosia 17: 55-62.

59. Zhu XD, Zhang QH, Kong LB, Wang F, Luo SD (2010) New hydroquinone diglycoside acyl esters and sesquiterpene and apocarotenoid from Ecdysanthera rosea. Fitoterapia 81: 906-909.

60. Ding L, Maier A, Fiebig HH, Lin WH, Peschel G, et al. (2012) Kandenols A-E, eudesmenes from an endophytic Streptomyces sp. of the mangrove tree Kandelia candel. Journal of Natural Products 75: 2223-2228.

61. Gong HQ, Wu QX, Liu LL, Yang JL, Wang R, et al. (2011) Sesquiterpenoids from the aerial parts of Inula japonica. Helvetica Chimica Acta 94: 1269-1274.

62. Liu ZL, Liu Q, Tian X (2007) The sesquiterpenes from Cacalia tangutica. Bulletin of the Korean Society 28: 292-302.

63. Arciniegas A, Pérez-Castorena AL, Reyes S, Contreras JL, Romo de Vivar A (2003) New oplopane and eremophilane eerivatives from Robinsonecio gerberifolius. Journal of Natural Products 66: 225-229.

64. Lu T, Vargas D, Fischer NH (1993) Sesquiterpenes from Brintonia discoidea. Phytochemistry 34: 737-743.

65. Jakupovic J, Ellmauerer E, Jia Y, Bohlmann F, Dominguez XA, et al. (1987) Further eudesmane derivatives from Verbesina species. Planta Medica 53: 39-44.

66. Rojatkar SR, Puranik VG, Tavale SS, Guru Row TN, Nagasampagi BA (1987) Eudesmane derivatives from Epaltes divericata. Phytochemistry 26: 569-570.

67. Kato T, Frei B, Heinrich M, Sticher O (1996) Sesquiterpenes with antibacterial activity from Epaltes mexicana. Planta Medica 62: 66-71.

68. Ahmad VU, Fizza K (1987) Arguticin, a new sesquiterpene from Pluchea arguta. Justus Liebigs Annalen der Chemie 7: 643-644.

69. Jakupovic J, Misra LN, Chau Thi TV, Bohlmann F, Castro V (1985) Cuauthemone derivatives from Tessaria integrifolia and Pluchea symphytifolia. Phytochemistry 24: 3053-3055.

70. Guilhon GMSP, Muller AH (1996) Eudesmane derivatives from Pluchea quitoc. Phytochemistry 43:417-421.

71. Jakupovic J, Ganzer U, Pritschow P, Lenmann L, Bohlmann F, et al. (1992) Sesquiterpene lactones and other constituents from Ursinia species. Phytochemistry 31: 863-880.

72. Marco JA (1989) Sesquiterpene lactones from Artemisia herba-alba subsp. herba-alba. Phytochemistry 28: 3121-3126.

73. Marco JA, Sanz JF, Yuste A, Carda C, Jakupovic J (1991) Sesquiterpene lactones from Artemisia inculta. Phytochemistry 30:3661-3668

74. Marco JA, Sanz JF, Jakupovic J, Huneck S (1990) New sesquiterpene lactones and acetylenes from Chrysanthemum lavandulifolium. Tetrahedron 46: 6931-6938.

75. Jakupovic J, Tan RX, Bohlmann F, Boldt PE, Jia ZJ (1991) Sesquiterpene lactones from Artemisia ludoviciana. Phytochemistry 30: 1573-1582.

76. Wang XX, Lin CJ, Jia ZJ (2006) Triterpenoids and Sesquiterpenes from Mulgedium tataricum. Planta Med 72: 764-767. [Crossref]

77. Zhang QF, Luo SD, Wang HY (1998) Two new sesquiterpenes from Atractylodes macrocehpala. Chinical and Chemistry Letters 9: 1097-1106.

78. Bohlmann F, Zdero C (1982) Sesquiterpene lactones and other constituents from Tanacetum parthenium. Phytochemistry 21: 2543-2548.

79. Hewlett MJ, Begley MJ, Groenewegen WA, Heptinstall S, Knight DW, et al. (1996) Sesquiterpene lactones from feverfew, Tanacetum parthenium: isolation, structural revision, activity against human blood platelet function and implications for migraine therapy. Journal of Chemical Society, Perkin Trans 22: 1979-1984.

80. Kastner U, Breuer J, Glasl S, Baumann A, Robien W, et al. (1995) Guaianolideendoperoxide and monoterpene-hydroperoxides from Achillea nobilis. Planta Medica 61: 83-88.

81. Zdero C, Bohlmann F (1989) Sesquiterpene lactones and other terpenes from Geigeria species. Phytochemistry 28: 3105-3111.

82. Ahmed AA, Jakupovic J, Bohlmann F, Regaila HA, Ahmed AM (1990) Sesquiterpene lactones from Xanthium pungens. Phytochemistry 29: 2211-2215.

83. Hausen BM, Breuer J, Weglewski J, Rücker G (1991) a-Peroxyachifolid and other new sensitizing sesquiterpene lactones from yarrow (Achillea millefolium L., Compositae) Contact Dermatitis 24: 274-280. 
84. Appendino G, Gariboldi P, Ano GM (1982) Crispolide, an unusual hydroperoxysesquiterpene lactone from Tanacetum vulgare. Phytochemistry 21: 10991102.

85. Gören N, Tahtasakal E, Pezzuto JM, Cordell GA, Schwartz B, et al. (1994) Sesquiterpene lactones from Tanacetum argenteum. Phytochemistry 36: 389-303.

86. Öksüz S, Serin S, Topçu G (1994) Sesquiterpene lactones from Centaurea hermannii. Phytochemistry 35: 435-441.

87. Bulatovic V, Vajs V, Macura S, Juranic N, Milosavljevic S (1997) Highly oxygenated guaianolides from Anthemis carpatica. Journal of Natural Products 60: 1222-1227.

88. Nagashima F, Suzuki M, Takaoka S, Asakawa Y (1998) New sesqui- and diterpenoids from the Japanese liverwort Jungermannia infusca (MITT.) STEPH. Chem Pharm Bull 46: 1184-1191.

89. Nagashima F, Suzuki M, Takaoka S, Asakawa Y (1999) New acorane-and cuparanetype sesqui-and new labdane-and seco-labdanne-type diterpenoids from the Japanese liverwort Jungermannia infusca (Mitt.) Steph Tetrahedron 55: 9117-9121.

90. Chokpaiboon S, Sommit D, Bunyapaiboonsri T, Matsubara K, Pudhom K (2011) Antiangiogenic effect of chamigrane endoperoxides from a Thai mangrove-derived fungus. Journal of Natural Products 74: 2290-2299.

91. Chokpaiboon S, Sommit D, Teerawatananond T, Muangsin N, Bunyapaiboonsri $\mathrm{T}$, et al. (2010) Cytotoxic nor-chamigrane and chamigrane endoperoxides from a basidiomycetous fungus. Journal of Natural Products 73: 1005-1012.

92. Efange SMN, Brun R, Wittlin S, Connolly JD, Hoye TR, et al. (2009) Okundoperoxide, a bicyclic cyclofarnesylsesquiterpene endoperoxide from Scleria striatinux with antiplasmodial activity. Journal of Natural Products 72: 280-283.

93. Ma WH, Tan CM, He JC, Duan PS, Qin LP (2011) A novel eudesmene sesquiterpenoid from Schisandra sphenanthera stems. Chemistry of Natural Compounds 47: 713-717.

94. Adio AM, König WA (2005) Sesquiterpene constituents from the essential oil of the liverwort Plagiochila asplenioides. Phytochemistry 66: 599-609. [Crossref]

95. Nagashima F, Matsumura N, Ashigaki Y, Asakawa Y (2003) Chemical constituents of the liverworts Bryopteris filicina, Plagiochila asplenioides and Porella canariensis. $J$ Hattori Bot Lab 94: 197-204.

96. Zhao X, Zheng GW, Niu XM, Li WQ, Wang FS, et al. (2009) Terpenes from Eupatorium adenophorum and their allelopathic effects on Arabidopsis seeds germination. Journal of Agriculture and Food Chemistry 57: 478-482.

97. Wang CF, Zhao Y, Liu YZ, Zhang ZZ (2009) Occurrence and biological activities of eremophilane-type sesquiterpenes. Chemical Research in Chinese Universities 25: 480-484.
98. He L, Hou J, Gan M, Shi J, Chantrapromma FS, et al. (2008) Cadinane sesquiterpenes from the leaves of Eupatorium adenophorum. Journal of Natural Products 71: 14851488 .

99. Müller S, Murillo R, Castro V, Brecht V, Merfort I (2004) Sesquiterpene lactones from Montanoa hibiscifolia that inhibit the transcription factor NF-?B. Journal of Natural Products 67: 622-630.

100. Mahmoud AA (1998) Xanthanolides and xanthane epoxide derivatives from Xanthium strumarium. Planta Medica 64: 724-727.

101. Rustaiyan A, Nahrevanian H, Kazemi M, Larijani K (2007) A new antimalarial agent effects of extracts of Artemisia diffusa against Plasmodium berghei. Planta Medica 73: 892-902.

102. Takaya Y, Kurumada KI, Takeuji Y, Kim HS, Shibata Y, et a. (1998) Novel antimalarial guaiane-type sesquiterpenoids from Nardostachys chinensis roots. Tetrahedron Lett 39: 1361-1364.

103. Takaya Y, Takeuji Y, Akasaka M, Nakagawasai O, Tadano T, et al. (2000) Novel guaiane endoperoxides, nardoguaianone A-D, from Nardostachys chinensis roots and their antinociceptive and antimalarial activities. Tetrahedron 56: 7673-7678.

104. Aguilar-Guadarrama AB, Rios MY (2004) Three new sesquiterpenes from Croton arboreous. Journal of Natural Products 67: 914-917.

105. Dong JY, Ma XY, Cai XQ, Yan PC, Yue L, et al. (2013) Sesquiterpenoids from Curcuma wenyujin with anti-influenza viral activities. Phytochemistry 85: 122-128.

106. Todorova M, Vogler B, Tsankova E (2000) Terpenoids from Achillea setacea. Z Naturforsch 55: 840-845.

107. Mohamed-Elamir F, Nakamura ES, Tawfik WA, Abdel-Azim NS, Abdel-Lateff A et al. (2015) Rare hydroperoxyl guaianolide sesquiterpenes from Pulicaria undulata. Phytochemistry Letters 12: 177-181.

108. Sy LK, Brown GD, Haynes R (1998) A novel endoperoxide and related sesquiterpenes from Artemisia annua which are possibly derived from allylic hydroperoxides. Tetrahedron 54: 4345-4356.

109. Sy LK, Ngo KS, Brown GD (1999) Biomimetic synthesis of arteannuin $\mathrm{H}$ and the 3,2-rearrangement of allylic hydroperoxides. Tetrahedron 55: 15127-15140.

110. Alza NP, Murray AP (2016) Chemical constituents and acetylcholinesterase inhibition of Senecio ventanensis Cabrera (Asteraceae). Record of Natural Products 10: $513-518$.

111. Rustaiyan A, Faridchehr A, Bakhtiyar M (2017) Sesquiterpene lactones of Iranian Compositae family (Astraceae); their chemical constituents and anti-plasmodial properties of tehranolide. Orient Journal of Chemistry 33.

Copyright: @2019 Siddiq A. This is an open-access article distributed under the terms of the Creative Commons Attribution License, which permits unrestricted use, distribution, and reproduction in any medium, provided the original author and source are credited. 